\title{
Selbstlernende Programme als Verantwortungsdilemma ${ }^{1}$
}

\author{
Jens Crueger und Thomas Krämer-Badoni
}

Im Jahr 2015 publizierte eine Gruppe von Wissenschaftlern und Wissenschaftlerinnen ein „Digital-Manifest“, das sich mit den Risiken der zunehmenden Nutzung von Algorithmen in allen Bereichen des Lebens auseinandersetzte. Dort skizzierten sie die folgende abschreckende Vision:

Angenommen, es gäbe eine superintelligente Maschine, die quasi gottgleiches Wissen und übermenschliche Fähigkeiten hätte - würden wir dann ehrfürchtig ihren Anweisungen folgen? Das erscheint durchaus möglich. Aber wenn wir das täten, dann hätten [...] Computer [...] die Kontrolle über die Welt übernommen. ${ }^{2}$

Diese Vision ist bislang noch nicht Realität geworden, aber tatsächlich steuert die Gesellschaft zunehmend auf einen solchen Zustand hin. Die Diskussion über Algorithmen, also von Menschen programmierte Entscheidungsroutinen und deren Fähigkeit, auf Grundlage gesammelter Erfahrungen hinzuzulernen und dadurch selbstständig Entscheidungen zu treffen, ist längst in der breiten Öffentlichkeit angekommen, wie ein Blick in die seriösen Presseorgane der letzten Jahre lehrt. Die Sorge, den unkontrollierbaren Entscheidungen einer selbstdenkenden Maschine ausgeliefert zu sein, beunruhigt immer mehr Menschen.

Im Folgenden erläutern wir zunächst, was Algorithmen sind und wie sie funktionieren. Dabei unterscheiden wir zwischen den Algorithmen, die ihre Berechnungen nur mit den eingegebenen Daten und entsprechend der vorformulierten Rechenanweisungen durchführen, und den selbstlernenden Algorithmen, die aus einer Vielzahl von zur Verfügung stehenden Daten neue Zusammenhänge herstellen und somit neue Wege zur Errei-

1 Wir danken der Informatikerin Prof. Dr. Kerstin Schill, Rektorin des Hanse-Wissenschaftskollegs, die sich die Zeit genommen hat, mit uns unsere Fragen zu Algorithmen zu diskutieren. Eventuelle Fehler sind selbstverständlich unsere Fehler, wie auch unsere Thesen Ergebnisse unseres Denkens sind.

2 Helbing et al. (17.11.2015). Die Autorinnen und Autoren dieses Manifestes sind: Dirk Helbing, Bruno S. Frey, Gerd Gigerenzer, Ernst Hafen, Michael Hagner, Yvonne Hofstetter, Jeroen van den Hoven, Roberto V. Zican und Andrej Zwitter. 
chung der vorgegebenen Ziele entwickeln. ${ }^{3}$ Danach werden wir an einigen Beispielen erläutern, welche ,Fehler ${ }^{6}$ in der Anwendung von Algorithmen auftreten können, woran das liegt und welche Folgen solche Fehler haben können.

Erst nach dieser Diskussion kann die Frage nach der Verantwortung für Fehler gestellt werden, die durch den Einsatz von Algorithmen zustande kommen. Dabei geht es um die Frage, wem bei der Anwendung von Algorithmen auftretende Fehler zurechenbar sind. Inwieweit kann die Verantwortlichkeit für die Entscheidungen der Algorithmen und deren Konsequenzen institutionalisiert werden? Die Gesellschaft wird eine Form finden müssen, Verantwortung für die Ergebnisse algorithmischer Operationen personell und/oder institutionell zuzuordnen und Verantwortlichkeiten zu benennen.

\section{Algorithmen}

Algorithmen sind von Menschen konstruierte mathematische Entscheidungsmodelle, die inzwischen in nahezu allen gesellschaftlichen Bereichen eingesetzt werden. Ein Algorithmus ist die mathematische Fassung eines Entscheidungsmodells: Wenn a dann b, und wenn a+ dann c. Die Mathematikerin Cathy O’Neil schreibt:

Ein Modell ist letztlich nichts anderes als eine abstrakte Simulation eines wie auch immer gearteten Prozesses, [...] es nimmt das vorhandene Wissen und nutzt es, um in diversen Situationen die entsprechenden Reaktionen vorherzusagen. Jeder Mensch trägt in seinem Kopf tausende Modelle mit sich herum. Sie sagen ihm, was er zu erwarten hat, und sie lenken seine Entscheidungen. ${ }^{4}$

Was passiert nun im Prozess der Mathematisierung solcher Entscheidungsmodelle? Zuerst muss man eine Vorstellung davon haben, welches Wissen man für eine Entscheidung in einem bestimmten Bereich braucht. Nehmen wir als Beispiel die Kreditvergabe durch Banken. ${ }^{5}$ Eine Privatperson

3 Eine ausgezeichnete und sehr gut verständliche Einführung in die verschiedenen Typen von Algorithmen hat vor kurzem die britische Mathematikerin Hannah Fry (2019) vorgelegt.

4 O’Neil (2017: S. 30).

5 Die Kreditvergabe eignete sich schon vor der Entwicklung von Algorithmen für die Analyse sozialer Chancenungleichheit, vgl. z. B. Jackson (1985). Seit solche Entscheidungen von Algorithmen unterstützt werden, findet sich die Kreditverga- 
geht zu ihrer Bank, um einen Kredit für den Kauf eines Hauses aufzunehmen. Die Bank will nun, bevor sie diesen Kredit möglicherweise bewilligt, bestimmte Dinge über diese Privatperson wissen, denn sie möchte das Risiko eines Kreditausfalls bestmöglich einschätzen können. Für diese Einschätzung helfen der Bank alle Informationen, die ihrer Erfahrung nach Aufschluss über die Rückzahlungsfähigkeit geben könnten, also z. B. die Höhe des Einkommens, die Art der beruflichen Tätigkeit, der Wert des zu kaufenden Hauses, frühere Kredite und deren Rückzahlung, aber auch andere Daten, die unter Umständen nicht so klar in Beziehung zur Rückzahlungsfähigkeit stehen. Dies sind etwa Alter, Alkoholkonsum, Gesundheit, Familienverhältnisse und weitere Informationen. Auch in den sozialen Medien und anderswo gesammelte Daten können einfließen. Die Bank speist diese personenbezogenen Daten in ihren Algorithmus ein, der bereits mit den Daten ihrer bisherigen Kreditnehmerinnen trainiert wurde. Der Algorithmus lernt also, welche Daten er berücksichtigen soll, und kann dann auf der Grundlage der eingespeisten Daten eine Berechnung durchführen, die der Bank als Entscheidungshilfe dient. Für die Bank stellt sich die Berechnung des Algorithmus' als umfassendes Erfahrungswissen dar: Der Algorithmus berechnet mit Hilfe der personenbezogenen Daten der antragstellenden Person und im Vergleich mit den bisherigen Kreditnehmerinnen der Bank, wie hoch die Wahrscheinlichkeit für eine Rückzahlung des Kredites ist. ${ }^{6}$

Fry nennt diesen Typus von Algorithmus den ,regelbasierten Algorithmus', den sie folgendermaßen charakterisiert:

Die Anweisungen werden von einem Menschen erstellt, sie sind direkt und eindeutig. Man kann sich das so vorstellen, dass diese Algorithmen der Logik eines Backrezepts folgen. Schritt eins: Tu dies. Schritt zwei: Wenn dies, dann das. Das bedeutet nicht, dass diese Algorithmen simpel sind - mit diesem Paradigma kann man jede Menge sehr leistungsfähige Programme erstellen. ${ }^{7}$

Die Mathematisierung und Digitalisierung des Entscheidungsprozesses sind mit dem vergleichbar, was auch in der Soziologie bei jedem quantitativen empirischen Verfahren passiert: Es gibt auf jeden Fall am Ende ein errechnetes Ergebnis. In der empirischen Soziologie werden theoretisch

be in vielen Texten, die auf die Problematik algorithmischer Entscheidungen aufmerksam machen wollen.

6 Vgl. Korczak/Wilken (15.01.2008).

7 Fry (2019: S. 23). 
definierte Zusammenhänge gemessen und dann in Aussagen folgenden Typs formuliert: Das in der Theorie erwartete Ergebnis wird unter bestimmten definierten Voraussetzungen mit einer Wahrscheinlichkeit von $\mathrm{x}$ Prozent eintreten. Ein Berechnungsergebnis ist auf jeden Fall da, zunächst einmal unabhängig davon, ob dieses Ergebnis das Kriterium der Signifikanz erfüllt oder nicht. Im Unterschied dazu ist das Ergebnis des Algorithmus ein Messwert, der auf der Grundlage der vermuteten logischen Beziehung vieler verschiedener theoretisch definierter Zusammenhänge zustande kommt. Dieser Wert wird im obigen Beispiel von der Bank als ein Ja oder ein Nein zur Kreditvergabe interpretiert. Vor der Einführung digitalisierter Entscheidungshilfen analysierten Bankangestellte so etwas selbst und entschieden mit Hilfe weniger Daten und vermutlich primär auf Basis der persönlichen Kenntnis der Bankkundin oder des Antragstellers. Die zuständigen Beschäftigten der Bank haben also gewissermaßen eine alltagstheoretisch begründete Wahrnehmung der Kundin in ihren Köpfen entwickelt. Die Algorithmen, mit so vielen Daten wie möglich gefüttert, betreiben nunmehr solche Entscheidungsverfahren als Rechenoperationen, die so komplex sind, dass die dahinter stehenden theoretischen Begründungen und logischen Zusammenhänge selbst bei den einfachen regelbasierten Algorithmen nicht ohne weiteres rekonstruiert werden können. Wie kommt es dazu? Fry erläutert das folgendermaßen:

Regelbasierte Algorithmen sind einfacher [als selbstlernende Algorithmen; Anm. J.C./T.K.B.] zu verstehen, weil die Anleitungen von Menschen geschrieben werden. Theoretisch kann jeder die Anweisungen einsehen und verstehen, auf welcher Logik die Abläufe in der Maschine basieren. ${ }^{8}$

Und in einer sehr aufschlussreichen Fußnote zu dieser Textstelle erläutert sie, weshalb sie hier „theoretisch kann jeder die Anweisungen [...]“ geschrieben hat:

Ich schreibe absichtlich ,theoretisch'. Die Realität sieht etwas anders aus. An manchen Algorithmen haben im Laufe der Jahre Hunderte oder gar Tausende Entwickler gearbeitet, und jeder hat eigene Schritte hinzugefügt. Mit der Zeilenanzahl des Programmcodes nahm auch die Komplexität des Systems zu, bis die Logikstränge so verwickelt waren wie ein Teller Spaghetti. Am Ende ist der Algorithmus nicht mehr

8 Ebd. (S. 24). 
nachvollziehbar und viel zu kompliziert, als dass ein Mensch ihn verstehen könnte. ${ }^{9}$

Man nennt dies auch die „Long Chain of Responsibility“10: Der Algorithmus ist zu einer Blackbox geworden.

Die Mathematisierung der Entscheidung - das können wir jetzt ganz allgemein zunächst für alle regelbasierten Algorithmen formulieren - bewirkt aber noch etwas anderes: Algorithmen gelten als objektiv und unfehlbar, die mathematischen Berechnungen sind ,theoretisch' (faktisch aber eben oft nicht) nachprüfbar, wiederholbar und kommen mit den gleichen Werten immer zu den gleichen Ergebnissen. Tatsächlich erzeugt aber nicht die Mathematik die Ergebnisse, sondern das Zusammenspiel der eingegebenen Werte mit den Handlungsanweisungen. Beides wird von Menschen eingegeben, aufgrund von Entscheidungen, die diese Menschen treffen. Die Mathematik setzt diese Anweisungen regelkonform um, die Personen verschwinden hinter dem mathematischen Regeln, man verliert sie regelrecht aus dem Blick. Der Algorithmus ist also keineswegs neutral und objektiv, sondern implizit interessengeleitet, standortgebunden und perspektivisch verzerrt. Es stellen sich dabei die Fragen, welche Informationen vom Algorithmus überhaupt verarbeitet und wie diese gewichtet werden? Welcher Ausschnitt der Realität wird erfasst und zu ,logischen Wahrheiten' verarbeitet? Ein Algorithmus ist und kann stets nur ein komplexitätsreduzierter Ausschnitt der Welt sein. Die Mathematik maskiert die an individuellen Interessen orientierte Subjektivität des Modells, das Ergebnis der Rechenoperation erscheint objektiv. Die von der Entscheidung Betroffenen haben deshalb in der Regel keine Möglichkeit, gegen die Ergebnisse des Algorithmus vorzugehen.

Anders als bei den regelbasierten Algorithmen entziehen sich die selbstlernenden Algorithmen - auch die unter Kontrolle selbstlernenden Algorithmen - der Rekonstruktion. Nehmen wir an, der Algorithmus hat alle personenbezogenen Daten aller deutschen Facebook- oder Google-Nutzenden zur Verfügung und soll diese Masse an Personen auf der Grundlage von Zusammenhängen der Daten in zehn Personengruppen einteilen. Das wird der Algorithmus lösen. Aber er wird nicht unterscheiden können, welche Zusammenhänge statistisch relevant und welche nur zufällig sind. Da der Algorithmus die Kriterien für die Auswahl der Zusammenhänge

9 Ebd. (S. 242, Anm. 13).

10 Wolfangel (03.05.2016). 
selbst entwickelt, entstehen hier willkürlich zusammengestellte Gruppen. ${ }^{11}$

Dies liegt vor allem an zwei Dingen: Zum ersten daran, dass diese Algorithmen mit sehr vielen Daten gefüttert werden. Je größer die Datenmenge, desto höher das Risiko, fehlerhafte Daten zu verwenden. ${ }^{12}$ Zum zweiten aber auch an der technischen Komplexität von Algorithmen. Julia Krüger verweist darauf, dass es sehr schwierig sei, Informationen über die maschinellen Entscheidungen zugrunde liegende Logik herauszuarbeiten. Und sie fährt fort: „Einer Transparenz von Algorithmen können unterschiedliche Dinge im Weg stehen, dazu zählt auch technische Komplexität."13

Was können, was sollten wir an dieser Stelle festhalten? Schon einfache Algorithmen sind so komplexe Systeme, dass der Nachvollzug der Entscheidungslogik der Maschine kaum noch möglich ist. Bei selbstlernenden Algorithmen ist letztendlich weder eine interne noch eine externe Überwachung bzw. Überprüfung möglich, jedenfalls nicht in einem Zeitraum, in dem über die Frage der Anwendung solcher Algorithmen angemessen entschieden werden könnte. Deshalb wird es letztlich darauf ankommen zu entscheiden, in welchen Entscheidungssituationen wir solche Algorithmen überhaupt zulassen wollen und wie mit Fehlern von algorithmenbasierten Entscheidungsprozessen umgegangen werden kann.

\section{Algorithmen und ibre Febler}

Unzweifelhaft begehen Algorithmen Fehler, aber es sind keine Fehler in der Berechnung, sondern Fehler, die auf das menschliche Handeln in und mit dem Algorithmus zurückgeführt werden müssen. Wenn ein Bilderkennungs-Algorithmus schwarze Menschen als ,Affen' (so geschehen beim Fotodienst Flickr) oder als ,Gorilla' (so geschehen bei Google) identifiziert, ${ }^{14}$ dann ist bei der Programmierung oder im Trainingsprogramm ein klarer Fehler entstanden, der einen rassistischen Bias offenbart. Die Risiken solcher Fehler sind bei Algorithmen mit komplexeren Aufgaben ungleich höher und zugleich schwerer zu erkennen, da der Zusammenhang zwischen

11 Vgl. auch Wolfangel (03.05.2016) und die dort aufgeführten Hinweise.

12 Vgl. ebd.

13 Krüger (19.01.2018).

14 Vgl. Drösser (2016: S. 208). 
den Ausgangsdaten und dem errechneten Ergebnis abstrakter und somit kaum extern nachprüfbar ist.

O'Neil berichtet, dass die Arbeitslosenversicherung von Michigan von 2013 bis 2015 einen Algorithmus eingesetzt hat, der zu katastrophalen Folgen führte:

Der Zweck dieses Algorithmus bestand darin, betrügerische Anträge auf Arbeitslosengeld zu finden, aber er lief dann völlig aus dem Ruder: er beschuldigte fälschlicherweise über 20000 Personen des Betrugs mit einer Falschtrefferquote von sage und schreibe 93 Prozent. Er hat das Leben zahlloser Menschen ruiniert, indem er ihnen Geldstrafen von bis zu 100000 Dollar aufbrummte, und zwar genau dann, als sie am dringendsten Geld brauchten. ${ }^{15}$

Der Zweck dieses Algorithmus - die Vermeidung von Betrug - ist natürlich ein legitimes Ziel. Aber zu Recht verweist O’Neil darauf, dass hier jegliche Form der Überprüfung des Algorithmus fehlte:

Man ignorierte dabei aber völlig die Möglichkeit, dass jemand ungerechtfertigterweise des Betrugs beschuldigt werden könnte. Obwohl es kaum Grund zu der Annahme gibt, dass hinter den falschen Treffern eine Absicht steckte, können sie auch nicht einfach nur als Folge eines technischen Fehlers abgetan werden. Die schlichte Tatsache, dass kein Verfahren der Rechenschaftslegung für dieses automatisierte System existierte, zeigt, dass kaum ein Gedanke an die potenziellen Opfer verschwendet wurde. ${ }^{16}$

In unseren Augen zeigt dieses Beispiel, welche gewaltigen Folgewirkungen aus solchen Fehlern resultieren können. Der Versuch, mit Hilfe des Algorithmus vermutlich wenige Fälle von Betrug zu klären, hat eine Vielzahl von Menschen ins Unglück gestürzt. Ein viel zu hohes Risiko.

Am bereits angesprochenen Beispiel der algorithmischen Entscheidung über die Kreditvergabe zeigt sich, dass eine algorithmische Entscheidung in zweifacher Hinsicht falsch sein kann: Einmal, indem sie an sich kreditwürdige Antragstellende als kreditunwürdig brandmarken kann, zum anderen dadurch, dass Antragstellende ohne hinreichende Bonität den $\mathrm{Zu}$ schlag für einen Kredit bekommen können. Bei Ja/Nein-Entscheidungen ist der Grat für eine richtige Entscheidung besonders schmal. Aus einer übergeordneten Sichtweise ist eine algorithmische Entscheidung, die auf

15 O’Neil (2016: S. 307 f.).

16 Ebd. (S. 308). 
eine Minimierung der Risiken für die Kreditgeber ausgerichtet ist, zugleich sozial und ökonomisch schädlich. Das sozial schädliche Potential ergibt sich daraus, dass gerade diejenigen, die am dringendsten einen Kredit benötigen, ausgeschlossen werden könnten. Das schädliche Potential auf ökonomischer Ebene entsteht vor dem Hintergrund, dass Kredite für die Entwicklung der Ökonomie eine zentrale Rolle spielen. Bei algorithmisch basierten Entscheidungen ist stets zu überprüfen, wessen Interesse im Entscheidungsprozess privilegiert wird. Die Vermutung liegt nahe, dass es meist das Interesse der Anwendenden ist, auf das hin der Algorithmus konstruiert sein dürfte.

Zwei weitere Beispiele weisen auf andere Folgewirkungen von Algorithmen im Kontext von Diskriminierung hin. Das erste Beispiel zeigt, dass wegen der Intransparenz der Entscheidungskriterien nicht festgestellt werden kann, ob es sich bei der Entscheidung des Algorithmus um eine Diskriminierung oder um eine Entscheidung handelt, in der die Diskriminierung bereits ausgesondert wurde.

Ein Algorithmus, insbesondere einer, der auf maschinellem Lernen beruht, tendiert [...] dazu, den Status quo zu zementieren. Er stellt dann zum Beispiel fest, dass Männer in Führungspositionen häufiger vertreten sind als Frauen, und präsentiert ihnen [den Männern; Anm. J.C./ T.K.B.] eher Jobanzeigen für Führungskräfte. [...] Weil die DeepLearning-Algorithmen ihre Regeln oft nicht explizit benennen können, ist es im Einzelfall schwierig, die Diskriminierung dingfest zu machen. ${ }^{17}$

Damit ersichtlich wird, ob die algorithmisierte Auswahl von potenziellen Angestellten das Ergebnis einer reproduzierten Diskriminierung ist, oder ob jener Diskriminierung im Algorithmus bereits entgegengewirkt wurde (aber beispielsweise mit dem Geschlecht korrelierende Variablen für die Diskriminierung verantwortlich sind), muss die Konstruktion des Algorithmus für Dritte transparent sein.

Beim zweiten Beispiel zeigt sich hingegen, dass es bei Fehlern auf beiden Seiten der Entscheidung schwer geschädigte Personen gibt. O’Neil berichtet von Algorithmen, die in vielen Städten der Vereinigten Staaten eingesetzt wurden, um das Risiko von Kindesmisshandlungen einzuschätzen. Von solchen Algorithmen geht ein sehr hohes beidseitiges Schädigungsri-

17 Drösser (2016: S. 223). Deep Learning oder neuronale Algorithmen sind Spezialfälle selbstlernender Algorithmen. Bei Drösser findet sich eine sehr gut verständliche Darstellung dieses Algorithmus-Typs. Vgl. ebd. (S. 197-211). 
siko aus: Einerseits im Hinblick auf Familien, die als Risiko in Bezug auf eine Misshandlung ihrer Kinder eingeschätzt werden, obgleich dieses Risiko eine Fehleinschätzung darstellen kann. Andererseits auch im Hinblick auf Familien, die nicht als Risiko für deren Kinder eingeschätzt werden, in denen es dann aber tatsächlich zur Misshandlung eines Kindes kommt. O’Neil fährt fort: „Wie können wir die beiden Fälle vergleichen? Die Antwort ist, dass wir sie vergleichen müssen, wenn wir den Algorithmus in geeigneter Weise konstruieren wollen. "18 Die Forderung nach einer Einsicht in Daten und Quellcode bei sensiblen, insbesondere normativ wirksamen algorithmischen Operationen geht einher mit der Notwendigkeit eines erweiterten Bewusstseins von Sozialempirie ${ }^{19}$ und Social Engineering ${ }^{20}$ im digitalen Zeitalter. Denn wo Algorithmen die Aufgabe verfolgen, soziale Problemlagen zu erkennen, zu bewerten und daraus Konsequenzen abzuleiten, müssen sie in dieser Rolle aus einer staatlichen Garantenstellung heraus besonders kritisch geprüft werden.

\section{Verantwortung}

Algorithmen verarbeiten personenbezogene Daten, die sie zu Handlungsempfehlungen jeder Art formen, sowohl für diejenigen, deren Daten sie verarbeiten, als auch für diejenigen, in deren Auftrag sie die Daten verarbeiten, also für die Anwendenden des Algorithmus. Im Folgenden geht es um die Fragen: Wer ist verantwortlich für den Schutz individueller Daten? Und: Wer ist verantwortlich für die Folgen fehlerhafter algorithmischer Entscheidungen?

Mit diesen Fragen ist allerdings auch eine sehr viel umfassendere Frage eng verknüpft, nämlich die nach der Verantwortung für die Erstellung und die Sicherung der für das Funktionieren der Digitalisierung notwendigen Infrastruktur. Diese Frage reicht - will man sie vollumfänglich beantworten - weit über die auf Algorithmen bezogenen Fragestellungen hinaus, aber sie muss zumindest in ihren Grundzügen genannt und beantwortet werden.

Beginnen wir mit dem vermeintlich ,einfachsten` Fall, dem Schutz personenbezogener Daten angesichts einer globalen Datenökonomie. Dafür

18 O'Neil (2017: S. 307).

19 Sozialempirie verstehen wir als das Erzeugen von Erkenntnissen über gesellschaftliche Zusammenhänge mittels datengetriebener Analyse.

20 Social Engineering verstehen wir im Sinne der Sozialtechnik als gezielte Bemühung zur Veränderung gesellschaftlicher Strukturen. 
sollte doch jedes Individuum selbst verantwortlich sein, schließlich erfolgt die Nutzung digitaler Technologien ja freiwillig. Aber wie steht es denn wirklich um diese Freiwilligkeit? Was geschieht, wenn jemand auf die Nutzung all dieser digitalen Infrastrukturen und Innovationen verzichtet? Aus was ist sie oder er dann ausgegrenzt? Und was würde der Verzicht auf die Nutzung der durch die Digitalisierung bestimmten Techniken für die heutigen Geschäftsmodelle und Wertschöpfungsketten bedeuten?

Betrachten wir zuerst die Unternehmen: Die Digitalisierung ist mehr als nur eine technische Entwicklung der Kommunikations- und Produktionsinstrumente. Sie verändert in häufig disruptiver Weise die bisherige Marktsituation, greift konventionelle Geschäftsmodelle an und verändert dabei das ökonomische System in nahezu jeder Branche. ${ }^{21}$ Insbesondere jene Zweige der Wirtschaft, die auf Vertrauen basieren - da sind neben dem Finanz- und Versicherungssektor beispielsweise die Gesundheits- und Sicherheitsbranche zu nennen -, benötigen seit jeher möglichst umfangreiche und verlässliche Informationen über ihre Vertragspartnerinnen. Die Algorithmisierung eröffnet diesen Branchen daher ein großes Wertschöpfungspotential, das sie kaum ungenutzt lassen dürften. ${ }^{22}$

Auf der Seite der Konsumierenden stellt die Digitalisierung mit ihren stetigen und extrem kurzen Innovationszyklen eine immense kulturelle Herausforderung dar. Die ,digitale Transformation ${ }^{623}$ ist mehr als eine neue Produktionstechnik, sie ist eine vollständige kulturelle Umwälzung der modernen Gesellschaft:

Kein Zweifel: die Zukunft nicht nur dieses Landes wird von Digitalisierungstechnologien geprägt sein. Das Internet hat in den vergangenen 20 Jahren bereits tiefgreifende Veränderung ausgelöst, aber das ist nichts im Vergleich zu dem, was sich in den nächsten 20 Jahren tun wird. Vernetzung und intelligente Systeme werden einen riesigen Wachstumsschub auslösen, von dem aber nur diejenigen profitieren werden, die rechtzeitig einen Gang hochgeschaltet und die sich bietenden Chancen ergriffen haben. ${ }^{24}$

Die radikale Umwälzung der Produktion wird eine ebenso radikale Veränderung der Verhaltensweisen zur Folge haben müssen, also eine Umwälzung der Arbeits- und Alltagskultur, aus der man sich individuell gar nicht

21 Vgl. beispielsweise Straubhaar (2019).

22 Vgl. auch Schweitzer/Peitz (2017).

23 Vgl. Cole (2015).

24 Ebd. (S. 15). 
loslösen kann, ohne sich selbst aus der Gesellschaft auszugrenzen. Unklar ist jedoch, wie genau sich diese Veränderung unserer Verhaltensweisen angesichts der Algorithmen gestalten wird. Damit nähern wir uns einer ersten Bestimmung der Frage: Verantwortung für was und Verantwortung von wem?

Die Nutzung digitaler Technologien zu rahmen ist wie bei allen anderen Infrastrukturen eine staatliche Aufgabe. Das Gemeinwesen muss dafür Sorge tragen, dass eine entsprechende Infrastruktur entsteht und funktionsfähig bleibt. Es ist zugleich gefordert, individuelle Informationsfreiheiten der Bürgerinnen zu garantieren. Auch die Aufsichtspflicht des Staates gegenüber Marktteilnehmenden erstreckt sich in die digitale Sphäre hinein und gewinnt am Beispiel der Algorithmen Kontur. Verfahren wie die Zertifizierung von Produkten oder die Qualitätsüberprüfung durch Akteure wie den TÜV können im Falle der Algorithmen Wege zur Erfüllung der staatlichen Garantenstellung weisen. ${ }^{25}$

In den USA zeigte sich schon vor Jahren, was passiert, wenn der Staat seine Bürgerinnen nicht davor schützt, von Unternehmen ob ihrer digitalen Spuren ausgenutzt zu werden. Firmen setzten dort im Zuge von Bewerbungsgesprächen die Anwärterinnen regelmäßig unter Druck, die $\mathrm{Zu}$ gangsdaten ihrer vertraulichen Social-Media-Profile preiszugeben, währenddessen sich der US-Kongress nicht auf einen wirksamen Schutz dagegen zu verständigen mochte. ${ }^{26}$ Die grundsätzliche und immer wieder neu zu stellende Frage lautet daher, in welchem Maße staatliche Regulierung und Aufsicht die Interessen der Nutzerinnen mit jenen der Unternehmen, insbesondere bezüglich der Wahrung von Geschäftsgeheimnissen und der globalen Wettbewerbsfähigkeit, miteinander in Einklang bringen kann. Davon wird die demokratische und freiheitliche Struktur von Gesellschaften ebenso wie die ökonomische Prosperität abhängen. Gelingt dieser fundamentale Interessenausgleich nicht, ist die Funktionsfähigkeit der demokratischen Gesellschaft in Frage zu stellen: Der Verzicht auf den Schutz individueller Daten untergräbt bzw. zerstört die Basisstruktur der Demokratie - die individuelle Freiheit. Die Nichtberücksichtigung fundamentaler unternehmerischer Interessen hätte hinsichtlich der Kompatibilität des freien Unternehmertums mit der Demokratie ebenso fatale Wirkungen.

25 Zur Governance-Perspektive siehe sehr eingehend Sauerwein (2018).

26 Vgl. Crueger (2013: S. 20 f.). 
Dies gilt, solange wir auf die westliche Vorstellung einer liberalen, auf der Freiheit des Individuums basierenden Demokratie bauen. ${ }^{27}$

Es war schon immer Aufgabe des Staates, für eine rechtliche Infrastruktur und die Schaffung eines Ausgleichs zwischen antagonistischen Interessen zu sorgen und es gibt zu dem, was wir heute erleben, eine passende Analogie aus dem 19. Jahrhundert: Während die süddeutschen Länder Bayern, Baden und Württemberg sowie Hannover, Braunschweig und Hessen-Kassel die Eisenbahnen schon von Anfang an als Staatsunternehmen organisierten, versuchten es einige nord- und ostdeutsche Länder mit einer staatlichen Aufsicht über die privat organisierten Eisenbahnunternehmen. Der Staat zwang Unternehmen, parallele Eisenbahnstrecken zu bauen und zu bedienen, was zu einer nahezu vernichtenden Konkurrenz führte, oder er zwang mehrere Eisenbahnunternehmen dazu, auf einer Strecke zu agieren. Beide Strategien führten zu Fusionen und so zur Entstehung eines privaten Monopols. In den 80er Jahren des 19. Jahrhunderts griff der Staat schließlich mit einer Verstaatlichung des Eisenbahntransportes durch. ${ }^{28}$ In unserem Kontext erinnert die Regulation der digitalen Infrastruktur an die Probleme der frühen Industrialisierung: Neue Technologien mit weitreichenden gesellschaftlichen Auswirkungen erfordern neue staatliche Setzungen.

Der Staat wird eine Lösung - also die Sicherung der Erstellung der digitalen Infrastruktur und deren Funktionsfähigkeit für alle gesellschaftlichen Akteure - herbeiführen müssen, und dafür tragen er und die privaten Unternehmen gemeinsam die Verantwortung. Es ist nicht abwegig, die grundlegenden Zuständigkeitsfragen zu stellen, denn es zeigt, dass die organisatorischen Probleme der Digitalisierung nicht ohne den Staat gelöst werden können. Denn die Digitalisierung ist nicht einfach nur eine weitere Entwicklungsstufe des Altbekannten, sie bedeutet vielmehr eine völlige Veränderung der Produktion und Kultur. Während sich die Grundstrukturen der Produktion bereits verändert haben und noch weiter verändern

27 Hier dürfte allerdings schon klar geworden sein, dass mit dem Staat unter der Voraussetzung der Globalisierung nicht mehr (oder nur partiell) der Nationalstaat gemeint sein kann. Da die Digitalisierung nicht an nationalstaatliche Grenzen und Regulierungen gebunden ist, wird die Regulierung zunehmend durch Staatenverbindungen wie die EU und/oder durch zwischenstaatliche Vereinbarungen stattfinden müssen. Das erschwert den für die Regulierung notwendigen Interessenausgleich zwischen den Staaten erheblich.

28 Vgl. Krämer-Badoni et al. (1971: S. 247-249.). Unser damaliger Überblick über die Eisenbahnpolitik der deutschen Länder ab den 30er Jahren des 19. Jhdts. basierte auf der Darstellung in Ritschl (1968: S. 31 f.). 
werden, wird deren kulturelle Umsetzung in Normen und Verhaltensweisen viel Zeit brauchen. Dieser Teil der Gesellschaft, die soziokulturelle Verankerung des Menschen mit seiner Wahrnehmung, seinen Gewohnheiten, seinen Normen und Erwartungen, ist sehr veränderungsresistent. Wie resistent er sein kann, zeigt übrigens ein Beispiel, das ebenfalls aus der Phase der frühen Industrialisierung stammt: Max Weber beschreibt in seiner ,Protestantischen Ethik', dass die Einführung der Akkordlöhne oft daran scheiterte, dass die Arbeitenden den Akkordlohn nicht dazu nutzten, mehr zu verdienen, sondern nur dazu, weniger zu arbeiten. Sie wollten nicht mehr verdienen, sondern weniger arbeiten, um das zu verdienen, was sie traditionell zum Leben brauchten. ${ }^{29}$ Was zeigt dieses Beispiel? Der entstehende Kapitalismus brauchte auch Menschen, die kapitalkonform dachten. Es zeigt, dass die kapitalistischen Strukturen sich die benötigten Mentalitäten und Denkweisen selbst erzeugen mussten. ${ }^{30}$ Und genau das gleiche geschieht heute durch die Techniken und die Infrastruktur der Digitalisierung. Die Mentalitäten, die eine digitalisierte Gesellschaft benötigt, müssen erst noch entstehen.

Die ,Algorithmisierung' der digitalisierten Gesellschaft wird durch viele Entwicklungen möglich, von denen in unserem Kontext zwei ein besonderes Gewicht haben: einerseits die ungeheure Zunahme der Rechen- und Datenspeicherungskapazitäten, zum anderen die Fähigkeit, die auf den großen und kleinen Plattformen gesammelten Daten - potenziell von nahezu der Gesamtzahl menschlicher Individuen - zu speichern und zu analysieren. Anders gesagt: Die Aufhebung der Privatheit ist die Voraussetzung für die Funktionsfähigkeit von Algorithmen. Wenn niemand seine privaten Daten zur Verfügung stellen würde, dann gäbe es nichts zu sammeln. Es geht dabei nicht nur um die freiwillig auf Social Media preisgegebenen Informationen, sondern auch und gleichgewichtig um die Spuren, die wir im Netz hinterlassen: Nummern, Zeiten, Bewegungsprofile usw. Damit sind wir an einem drastischen Widerspruch angelangt: Die digitale Gesellschaft braucht die Auflösung der individuellen Privatheit genauso

29 Vgl. Weber (1978: S. 30 ff., hier insbesondere S. 44 f.).

30 Max Weber sieht den Kapitalismus dezidiert als Akteur: „Überall, wo der moderne Kapitalismus sein Werk der Steigerung der ,Produktivität‘ der menschlichen Arbeit durch Steigerung ihrer Intensität begann, stieß er auf den unendlich zähen Widerstand dieses Leitmotivs präkapitalistischer wirtschaftlicher Arbeit, und er stößt auch heute überall um so mehr darauf, je ,rückständiger` (vom kapitalistischen Standpunkt aus) die Arbeiterschaft ist, auf die er sich angewiesen sieht." Ebd. (S. 45). 
dringend und unbedingt wie die Bürgerinnen Privatheit als unabdingbare Voraussetzung von Freiheit benötigen. ${ }^{31}$ Wie kann das zusammengehen?

\section{Resümee und Ausblick}

Nun sind wir an dem Punkt angelangt, an dem das eigentliche Dilemma beginnt und deutlich geworden sein sollte, dass es keine einfachen Antworten gibt auf die Frage: Wer kann angesichts von algorithmisierten Prozessen wofür verantwortlich gemacht werden?

Während wir uns Normen und Regelungen auszudenken versuchen, die uns zu einer angemessenen Bewältigung der durch die Digitalisierung aufgeworfenen Probleme leiten sollen, entwickelt sich die digitale Technik rasant fort. Kaum glauben wir, etwas begriffen zu haben und darauf angemessen reagieren zu können, schon sehen wir neue Probleme vor uns.

Diese Problemlage erfordert von uns allen Geduld und Gelassenheit, und die Überzeugung, dass wir geeignete Lösungen finden werden. Fangen wir also mit den Überlegungen an:

1. Privatheit, wie wir sie in der bürgerlichen Gesellschaft entwickelt und erlebt haben, wird es zukünftig nicht mehr geben. Das heißt nicht, dass gar keine Privatheit oder Privatsphäre mehr existieren wird. Die physische Sicherheit der Wohnung als Schutz- und Lebensraum wird aufrecht erhalten bleiben, Schutz vor dem Abhören von Telefonaten wird es weiterhin geben, eine vollständige Verschlüsselung wie z. B. bei WhatsApp wird ebenfalls vorhanden sein. Und doch wird es - je mehr wir unseren Alltag durch digitalisierte Instrumente bewältigen (Stichworte: Smart Homes und Smart Cities) - immer schwieriger werden, uns vor externen Eingriffen zu schützen. ${ }^{32}$ Keine Software, kein Be-

31 Es ist hier nicht der Ort, eine ausführliche Analyse von Privatheit und Privatsphäre vorzunehmen. Wir wollen aber daran erinnern, dass Privatheit ein junges (oder spätes) gesellschaftliches Produkt ist. Sie entsteht mit der bürgerlichen Gesellschaft und befindet sich wie alle Wertvorstellungen in einem kontinuierlichen Wandlungsprozess. Privatheit ist ebenso wenig ein festgefügtes Konzept wie Freiheit oder Sicherheit. Es wird immer darum gehen, das Verhältnis von Privatheit, Sicherheit und Freiheit unter den sich verändernden Bedingungen der digitalisierten Gesellschaft jeweils neu auszutarieren.

32 Die Zeitschrift brand eins hat im März 2019 dem Heft den folgenden Titel gegeben: „Alexa, sag' bitte meinem Kühlschrank, dass er meinem Fernseher sagen soll, dass er dem Rasenmäher sagen soll, dass er meine Smart-watch fragen soll, wie viel Uhr es ist. - Wo Digitalisierung im Alltag nützt" Vgl. brand eins (2019). Die- 
triebssystem und keine digitale Sicherheitsarchitektur kann dauerhaft für absolute Sicherheit sorgen. Hier muss eine Balance gefunden werden zwischen einer staatlichen Sicherung der digitalen Infrastruktur und einer individuell nutzbaren Sicherheitsarchitektur. Eine hundertprozentige Sicherheit wird und kann es nicht geben. Wir werden entscheiden müssen - als Personen und als Gesellschaft - wie viel Unsicherheit wir vertragen und wie viel Sicherheit wir verlangen können.

2. In einer digitalisierten Gesellschaft wird sich das Sammeln der Daten nicht verhindern lassen. Denn diese Daten entstehen und werden allein aufgrund ihres Vorhandenseins gesammelt. Wir halten es kaum für möglich, das Sammeln der Daten auch nur partiell einzuschränken, und eine Kontrolle darüber, wer die Daten analysiert, wird mit der exponentiell wachsenden Datenmenge immer schwieriger. ${ }^{33}$ Wir plädieren nicht für eine Aufgabe des Datenschutzes; wirksame Gesetze, die unsere Daten schützen, ohne unsere Freiheit einzuschränken, braucht es dringender denn je. Wir müssen uns insbesondere darüber verständigen, welche unserer Daten wir zum engsten Kreis unserer informationellen Selbstbestimmung zählen. Es geht dabei um eine gesellschaftliche Aushandlung, die unbeschadet der bisherigen gesetzgeberischen Bemühungen auf diesem Gebiet stattfinden muss. Vermutlich dürften dazu alle Daten gehören, die uns eine individuelle Lebensgestaltung innerhalb des gesellschaftlich zulässigen Verhaltensspektrums ermöglichen, für das wir nicht negativ sanktioniert werden wollen. Die Schlechterstellung von Personen, deren Lebensstil nicht den Vorstellungen von Krankenversicherungen über Gesundheitsprävention entspricht, ist in diesem Kontext ein virulentes Beispiel.

3. Die Funktionsfähigkeit der digitalisierten Gesellschaft basiert auf einer zumindest partiellen Auflösung, oder aber einer Transformation des Verständnisses von Privatheit. Dafür, dass die Individuen dies akzeptieren, muss ihnen ein Preis gezahlt werden. Zumindest dieser: Aus den Datensammlungen dürfen den Individuen keine Nachteile erwachsen, weder in privater noch in beruflicher Hinsicht. Wie eine solche Regelung formuliert und praktisch umgesetzt werden kann, ist offen. Aber wir glauben, dass der Schutz des Individuums vor den negativen Auswirkungen einer Verwendung seiner (auch freiwillig) veröffentlichten

ser satirisch gemeinte Titel kommt allerdings der Wirklichkeit sehr nahe: Je mehr wir unser Alltagsleben digital steuern, desto grenzenloser unsere Datenmengen, und desto unmöglicher auch die Kontrolle darüber, wer diese Daten wie nutzt.

33 Vgl. Krämer-Badoni (2018: S. 351-355). 
und/oder gesammelten Daten eine Mindestkompensation für den Verlust eines großen Teils der Privatsphäre darstellt. Ein notwendiger digitaler Gesellschaftsvertrag könnte wie folgt lauten: Sammelt und analysiert, forscht und entwickelt die künstliche Intelligenz, aber ihr habt darauf zu achten, mit den gesammelten Daten nicht den Personen zu schaden, mit deren Daten ihr arbeitet. Anders gesagt: Die Digitalisierung darf weder die Menschheit noch die individuelle Freiheit unterwerfen.

4. Und nun zum eigentlichen Ausgangspunkt unserer Überlegungen: dem Verantwortungsdilemma bei der Verwendung hochkomplexer Algorithmen. Waren wir ursprünglich davon ausgegangen, dass sich im Prinzip bei allen nicht auf Deep Learning (oder neuronalen Netzen) beruhenden Algorithmen die Verantwortung für das algorithmische Resultat feststellen lässt, so haben wir diese Einschätzung inzwischen relativieren müssen. Hierzu haben vor allem zwei unterschiedliche Erkenntnisse beigetragen, die wir bereits skizziert haben. Einerseits die Long Chain of Responsibility, die eine genaue Zuordnung von Verantwortung verhindert; zum anderen die hohe Komplexität der Algorithmen, die mit der wachsenden Datenmenge kontinuierlich zunimmt. Eine schnelle und sichere Rekonstruktion einer individuellen Verantwortung für ein schadenbringendes Ergebnis eines Algorithmus scheint letztlich nicht möglich zu sein, und zwar weder bei den nur komplexen, noch bei den auf neuronalen Netzen basierenden Algorithmen. In allen diesen Fällen wäre zu versuchen, durch die Einführung eines Ethikcodexes und diesem entsprechender Konstruktions-, Anwendungs- und Überprüfungsregeln die Fehlerquellen zu minimieren. Dort, wo dies nicht gelingt, wird es zu einer institutionellen Verantwortung kommen müssen. Und letztlich wird der Staat subsidiär die Verantwortung übernehmen müssen. Auch dies gehört zur Verantwortung des Staates gegenüber seinen Bürgerinnen.

5. Andere digitalethische Überlegungen gehen in Richtung einer professionsethischen Selbstverpflichtung, wie sie in der Medizin als Eid des Hippokrates sowie im Journalismus als Pressekodex etabliert sind. Dieser Weg, Verantwortung einerseits einem Kollektiv zuzuschreiben, das qua Tätigkeit in sensibler Stellung agiert, diese kollektiv übertragene Verantwortung dann aber auf die Handlungen des jeweiligen Individuums herunterzubrechen und eine Selbstverantwortung entlang berufsständisch definierter und kontrollierter Kriterien zu verlangen, mag auch für die Entwicklung und den Umgang mit Algorithmen funktionieren. Allerdings muss hier auch gesagt werden, dass die Formierung eines entsprechenden, dem Journalismus oder der Ärzteschaft korre- 
spondierenden Berufsverbandes, angesichts der Vielfältigkeit und Verzweigtheit der mit Algorithmen hantierenden Professionen sehr schwierig sein dürfte.

6. Wir wollen hier nochmals auf die Politikwissenschaftlerin Julia Krüger zurückkommen, die ihren 2018 unter dem Titel „Wie der Mensch die Kontrolle über den Algorithmus behalten kann" ${ }^{34}$ publizierten Text mit dem folgenden Absatz beendet:

Die Überantwortung menschlicher Entscheidungen an Maschinen steigert die gesellschaftliche Komplexität. Sie birgt ganz neue Herausforderungen gesellschaftlicher Herrschaftskontrolle. [...] Maschinelles Lernen und andere Formen künstlicher Intelligenz stellen ein ungeheuer spannendes und risikoreiches Experiment mit der Gesellschaft dar. [...] Es gilt genau jetzt, die Weichen zu stellen, wozu die Maschinen dem Menschen dienen sollen. ${ }^{35}$

Wir fürchten, dass die Ermöglichung einer Kontrolle über die Algorithmen, die ja bereits jetzt schon fast unmöglich ist, mit der Zunahme und Perfektionierung von unkontrolliert lernenden Algorithmen immer weniger möglich sein wird. Wir werden uns entscheiden müssen, welche gesellschaftlichen Bereiche künftig ohne maschinelles Lernen auskommen sollten.

7. Es sollte letztlich keinen rechtsfreien gesellschaftlichen Raum geben, in dem eine Kontrolle der Algorithmen und eine Rückführung von Fehlern auf individuelle Personen und/oder Institutionen als Verantwortliche nicht möglich ist. Sollte es sich jedoch herausstellen, dass eine solche Transparenz und Rekonstruierbarkeit herzustellen bei den komplexeren Algorithmen unmöglich bleibt, dann sollten solche Algorithmen nicht zur Anwendung kommen dürfen. Man kann entsprechend unserer Ausführungen in Punkt 6 entscheiden, dass Algorithmen in vielen Bereichen auch dann eingesetzt werden können, wenn sie nicht transparent sind, nämlich dort, wo sie keinen erheblichen Schaden anzurichten vermögen. In allen sensiblen Bereichen aber, wo Menschen nachhaltig geschädigt werden könnten, sollte die Anwendung intransparenter Algorithmen nicht zugelassen sein.

34 Krüger (19.01.2018).

35 Ebd. 


\section{Literaturverzeichnis}

brand eins (2019): Schwerpunkt: Digitalisierung. URL: https:/www.brandeins.de/ magazine/brand-eins-wirtschaftsmagazin/2019/digitalisierung [Abruf am: 11.11.2020].

Cole, Tim (2015): Digitale Transformation. Warum die deutsche Wirtschaft gerade die digitale Zukunft verschläft und was jetzt getan werden muss! München: Franz Vahlen.

Crueger, Jens (2013): „Privatheit und Öffentlichkeit im digitalen Raum. Konflikt um die Reichweite sozialer Normen“. In: Aus Politik und Zeitgeschichte (APuZ) 63 (15-16), S. 20-24.

Drösser, Christoph (2016): Total berechenbar? Wenn Algorithmen für uns entscheiden. München: Carl Hanser.

Fry, Hannah (2019): Hello World. Was Algorithmen können und wie sie unser Leben verändern. München: C.H. Beck.

Helbing, Dirk et al. (2015): „Das Digital-Manifest. Digitale Demokratie statt Datendiktatur“. In: Spektrum.de (17.11.2015). URL: https://www.spektrum.de/news/ wie-algorithmen-und-big-data-unsere-zukunft-bestimmen/1375933 [Abruf am: 13.09.2019].

Jackson, Kenneth T. (1985): Crabgrass Frontier: The Suburbanization of the United States. New York und Oxford: Oxford University Press.

Korczak, Dieter/Wilken, Michael (2008): „Scoring im Praxistest: Aussagekraft und Anwendung von Scoringverfahren in der Kreditvergabe und Schlussfolgerungen“. In: Verbraucherzentrale Bundesverband (15.01.2008). URL: https://www.v zbv.de/sites/default/files/mediapics/scoring_studie_15_01_2008.pdf [Abruf am: 10.04.2020].

Krämer-Badoni, Thomas (2018): „Social Media, Algorithmen und die urbane Kultur“. In: Gestring, Norbert/Wehrheim, Jan (Hrsg.): Urbanität im 21. Jahrhundert. Frankfurt am Main und New York: Campus, S. 344-362.

Krämer-Badoni, Thomas et al. (1971): Zur sozio-ökonomischen Bedeutung des Automobils. Frankfurt am Main: Suhrkamp.

Krüger, Julia (2018): „Wie der Mensch die Kontrolle über den Algorithmus behalten kann“. In: Netzpolitik.org (19.01.2018). URL: https://netzpolitik.org/2018/al gorithmen-regulierung-im-kontext-aktueller-gesetzgebung/ [Abruf am: 10.09.2019].

O’Neil, Cathy (2017): Angriff der Algorithmen: Wie sie Wahlen manipulieren, Berufschancen zerstören und unsere Gesellschaft gefährden. München: Carl Hanser.

Ritschl, Hans (1968): Vom Verkehrschaos zur Verkehrsordnung. Hamburg: Wegner.

Sauerwein, Florian (2018): „Automatisierung, Algorithmen, Accountability. Eine Governance Perspektive“. In: Rath, Matthias et al. (Hrsg.): Maschinenethik. Normative Grenzen autonomer Systeme. Wiesbaden: Springer VS, S. 35-56. 
Schneier, Bruce (2015): Data and Goliath. The Hidden Battles to Collect Your Data and Control Your World. New York und London: W.W. Norton \& Company.

Schweitzer, Heike/Peitz, Martin (2017): „Datenmärkte in der digitalisierten Wirtschaft. Funktionsdefizite und Regelungsbedarf“. In: ZEW Discussion Paper 17043. URL: http://hdl.handle.net/10419/170697 [Abruf am: 10.04.2020].

Straubhaar, Thomas (2019): Die Stunde der Optimisten. So funktioniert die Wirtschaft der Zukunft. Hamburg: Edition Körber.

Weber, Max (1978): „Die protestantische Ethik und der Geist des Kapitalismus“. In: Ders.: Gesammelte Aufsätze zur Religionssoziologie, Band I. Tübingen: Mohr Siebeck, S. 17-206.

Wolfangel, Eva (2016): „Die Grenzen der künstlichen Intelligenz“. In: Spektrum.de (03.05.2016). URL: https://www.spektrum.de/news/die-grenzen-der-kuenstliche n-intelligenz/1409149 [Abruf am: 10.09.2019]. 
